\title{
Constructive Genetic Algorithm for Clustering Problems
}

Luiz Antonio Nogueira Lorena

lorena@lac.inpe.br

LAC-Instituto Nacional de Pesquisas Espaciais, Av. dos Astronautas 1758 - Caixa

Postal 515, 12201-970 São José dos Campos-SP, Brazil

João Carlos Furtado

jcarlosf@dinf.unisc.br

Universidade de Santa Cruz do Sul, Av. Independência 2293, 96815-900 Santa Cruz do

Sul, Brazil

\begin{abstract}
Genetic algorithms (GAs) have recently been accepted as powerful approaches to solving optimization problems. It is also well-accepted that building block construction (schemata formation and conservation) has a positive influence on GA behavior. Schemata are usually indirectly evaluated through a derived structure. We introduce a new approach called the Constructive Genetic Algorithm (CGA), which allows for schemata evaluation and the provision of other new features to the GA. Problems are modeled as bi-objective optimization problems that consider the evaluation of two fitness functions. This double fitness process, called $f g$-fitness, evaluates schemata and structures in a common basis. Evolution is conducted considering an adaptive rejection threshold that contemplates both objectives and attributes a rank to each individual in population. The population is dynamic in size and composed of schemata and structures. Recombination preserves good schemata, and mutation is applied to structures to get population diversification. The CGA is applied to two clustering problems in graphs. Representation of schemata and structures use a binary digit alphabet and are based on assignment (greedy) heuristics that provide a clearly distinguished representation for the problems. The clustering problems studied are the classical p-median and the capacitated p-median. Good results are shown for problem instances taken from the literature.
\end{abstract}

Keywords

Genetic algorithms, clustering problems, p-median problems, capacitated p-median problem.

\section{Introduction}

Genetic algorithms (GAs) have been recognized as powerful approaches to solving optimization problems (Bäck and Schwefel, 1993; Davis, 1991; De Jong, 1975; Goldberg, 1989; Holland, 1975; Lorena and Lopes, 1996, 1997; Michalewicz, 1996; Mitchell, 1996). The foundation of such algorithms is the controlled evolution of a structured population.

The GA works on a set of variables called structures. When applying them to optimization problems, the first step is to define a coding scheme that allows a one-to-one mapping between solutions and structures. The following string can represent a structure $s_{k}=\left(s_{k 1}, s_{k 2}, \ldots, s_{k n}\right)$, where $n$ is the number of variables in the problem. A fitness function assigns a numeric value to each member of the current population (a collection of structures). Selection (like tournament or biased roulette wheel) is used together 
with crossover and mutation operators. The best structure is kept after a predefined number of generations (Goldberg, 1989; Holland, 1975; Michalewicz, 1996).

Holland (1975) put forward the Building Block Hypothesis (schema formation and conservation) as a theoretical basis for the GA mechanism. In his view, avoiding disruption of good schema is the basis for the good behavior of a GA. However, a major problem with building blocks is that schemata are evaluated indirectly via evaluation of their instances (structures). Goldberg and collaborators (Goldberg et al., 1989, 1993; Kargupta, 1995) introduced the messy-GA that allows variable length strings and looks for the construction and preservation of good building blocks.

The Constructive Genetic Algorithm (CGA) is proposed here as an alternative to the traditional GA approach (Holland, 1975), particularly in that CGA directly evaluates schemata. The population, initially formed only by schemata, is built, generation after generation, by directly searching for a population of well-adapted structures and also for good schemata.

Some steps in the CGA are notably different from a classical GA. The CGA works with a dynamic population, initially composed of schemata, which is enlarged after the use of recombination operators, or made smaller along the generations, guided by an evolution parameter. Schemata recombination diversifies the population thereby generating new schemata or structures. At the time of its creation, each schema or structure receives a rank used in the evolution analysis. Structures represent feasible solutions, undergo mutation, and are compared to the best solution found so far, which is always retained. Another main difference between a classical GA and a CGA is the new $f g$-fitness process.

The CGA will be explained in detail in Sections 2 and 3. We will explain the method with examples based on the clustering applications.

Clustering problems generally appear in classification of data for some purpose like storage and retrieval or data analysis. Any clustering algorithm will attempt to determine some inherent or natural grouping in the data, using distance or similarity measures between individual data (Spath, 1980; Zupan, 1982). In this paper, we use graphs to examine the application of CGA to two clustering problems: the classical p-median problem (PMP) and the capacitated p-median problem (CPMP).

The PMP is a classical location problem. The objective is to locate $p$ facilities (medians) so as to minimize the sum of the distances from each demand vertex to its nearest facility (Hakimi, 1964, 1965). The problem is well known to be NP-hard (Garey and Johnson, 1979), and several heuristics have been developed for p-median problems (Densham and Rushton, 1992; Goodchild and Noronha, 1983; Rolland et al., 1997; Rosing and ReVelle, 1997; Rosing et al., 1998; Teitz and Bart, 1968). More complete approaches explore a search tree (Beasley, 1993; Christofides and Beasley, 1982; Efroymson and Ray, 1966; Galvão and Raggi, 1989; Jarvinen et al., 1972; Neebe, 1978). Other approaches consider Lagrangian relaxation and subgradient optimization in a primaldual viewpoint (Beasley, 1993; Senne and Lorena, 2000).

The CPMP considers capacities for the service to be given by each median. The total service demanded by the vertices identified by p-median clusters cannot exceed the service capacity. Apparently, the CPMP was not as intensively studied as the classical PMP. Similar problems appeared in Klein and Aronson (1991), Maniezzo et al. (1998), Mulvey and Beck (1984), and Osman and Christofides (1994).

This paper is organized as follows. The CGA description is divided into two sections. In Section 2, we present aspects of modeling to be considered when solving a problem using CGA. Modeling involves definitions of the schema and structure rep- 


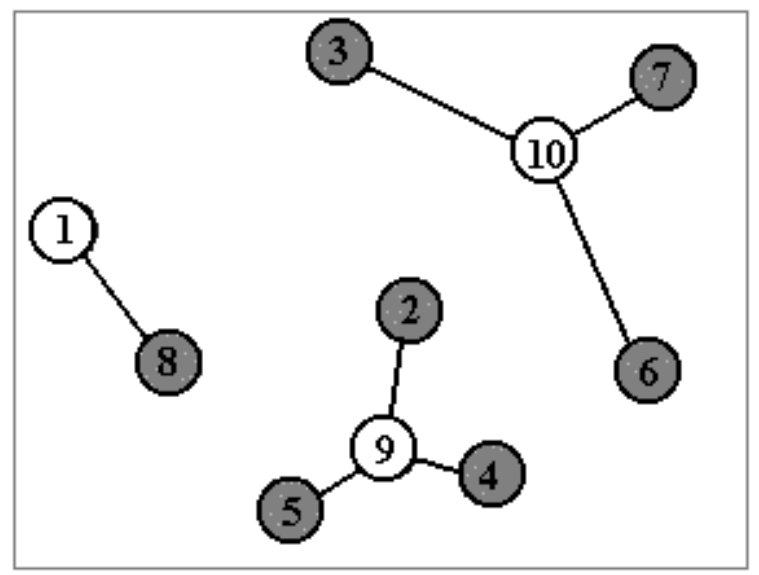

Figure 1: A 3-median solution.

resentations and the consideration of the problems at issue as bi-objective optimization problems. The evolution process is also described in this section. Section 3 describes the CGA operators: selection, recombination, and mutation, as well as the definition of an initial population and a CGA pseudocode. Section 4 shows computational results using instances from the literature. We conclude in Section 5 with a summary of the CGA performance and characteristics.

\section{CGA Modeling}

In this section, we describe the modeling phase of the CGA. The clustering problems are formulated as bi-objective optimization problems. Two fitness functions are defined on the space of all schemata and structures that can be obtained using a specific representation. The evolution process considers the two objectives on an adaptive rejection threshold, which gives ranks to individuals in the population and yields a dynamic population.

\subsection{Structure and Schema Representation}

Very simple structure and schema representations are adopted for PMP and CPMP. They use a binary alphabet, and assignment heuristics make a clear and independent connection with the two clustering problems. The use of the same kind of representation allows the remaining steps in CGA to be valid for both problems.

Suppose a given graph $G=(V, E)$. A clustering problem in graphs can be stated as the search for partitions on the vertex set $V$ in a (generally) predefined number of clusters, optimizing some measure on combinations of vertices and/or edge weights. The problems considered in this paper are clustering problems in graphs.

A typical problem instance is composed of $n$ demand points (vertices) $V=\{1, \ldots, n\}$ and a distance (weight) matrix $\left[\mu_{j l}\right]$ indicating distances between pairs of vertices such that $\mu_{j l} \geq 0, \mu_{j j}=0$, and $\mu_{j l}=\mu_{l j}$ for all $j, l \in V$.

To define the representation, some vertices are elected as the seeds, i.e., the initial vertices in clusters that in some way attract the other vertices that participate in the representation. 


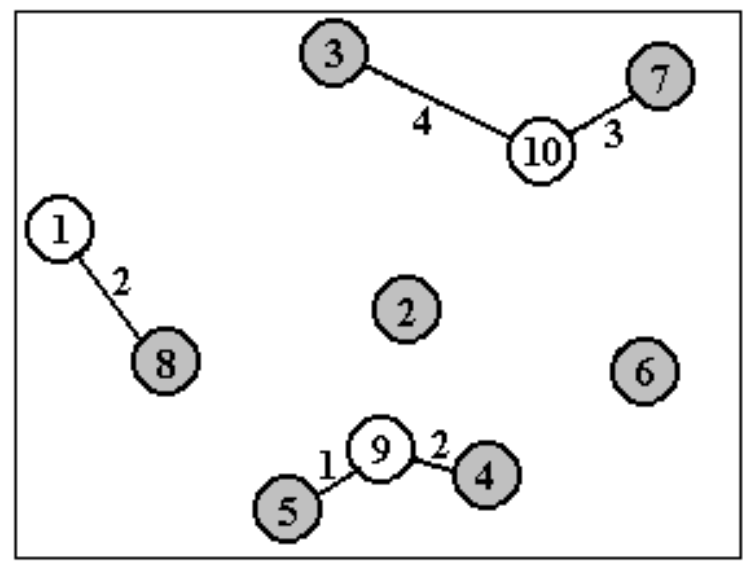

Figure 2: A 3-median schema.

Starting with an example for the PMP, consider the following 3-median solution for a complete graph $G(V, E)$ instance with 10 vertices (see Figure 1).

A partition on the index vertex set $V$ is then made, yielding two blocks, the seed set and the non-seed set, where the seeds are the medians. For the 3-median solution of Figure $1, V_{1}\left(s_{k}\right)=\{1,9,10\}$ is the seed set, and $V_{0}\left(s_{k}\right)=\{2,3,4,5,6,7,8\}$ is the nonseed set. Vertices 1,9 , and 10 are the medians, and the others are assigned to a median. The 3-median structure for the example will be $s_{k}=(1,0,0,0,0,0,0,0,1,1)$, where each position $s_{k j}$ in $s_{k}$, receiving labels 1 or 0 , means that vertex $j$ belongs to sets $V_{1}\left(s_{k}\right)$ or $V_{0}\left(s_{k}\right)$, respectively.

Structure $s_{k}$ is not completely defined, as we do not know the non-median assignments. For the clustering problems, after the initial seed identification, an Assignment Heuristic is employed to assign non-seed vertices to clusters. For the PMP, each non-median vertex is assigned to the nearest identified median. Algorithm AH1 (shown below) formalizes the assignments.

\section{AH1}

Read $s_{k}$,

$$
\begin{aligned}
& V_{1}\left(s_{k}\right)=\left\{\zeta_{1}, \zeta_{2}, \ldots, \zeta_{\left|V_{1}\left(s_{k}\right)\right|}\right\}, \\
& V_{0}\left(s_{k}\right)=\left\{\beta_{1}, \beta_{2}, \ldots, \beta_{\left|V_{0}\left(s_{k}\right)\right|}\right\} ;
\end{aligned}
$$

For $i=1$ to $\left|V_{1}\left(s_{k}\right)\right|$ do

end_for

$$
C_{i}\left(s_{k}\right):=\left\{\zeta_{i}\right\} ;
$$

For $j=1$ to $\left|V_{0}\left(s_{k}\right)\right|$ do

$$
\begin{aligned}
& r:=\operatorname{index}\left[\operatorname{Min}_{\left\{i=1, \ldots,\left|V_{1}\left(s_{k}\right)\right|\right\}}\left\{\mu_{\zeta_{i} \beta_{j}}\right\}\right] ; \\
& C_{r}\left(s_{k}\right):=C_{r}\left(s_{k}\right) \cup\left\{\beta_{j}\right\} ;
\end{aligned}
$$

end_for

After the application of AH1, exactly $p=\left|V_{1}\left(s_{k}\right)\right|$ clusters are identified (for the example in Figure $1, C_{1}\left(s_{k}\right)=\{1,8\}, C_{2}\left(s_{k}\right)=\{2,4,5,9\}$, and $\left.C_{3}\left(s_{k}\right)=\{3,6,7,10\}\right)$ 
corresponding to the median set $V_{1}\left(s_{k}\right)=\{1,9,10\}$.

The CGA works directly with schemata. A 3-median schema for the example can be $s_{k}=(1, \#, 0,0,0, \#, 0,0,1,1)$ as Figure 2 clarifies. The defined sets are: $V_{1}\left(s_{k}\right)=$ $\{1,9,10\}, V_{0}\left(s_{k}\right)=\{3,4,5,7,8\}$, and the new set $V_{\#}\left(s_{k}\right)=\{2,6\} . V_{\#}\left(s_{k}\right)=V-$ $\left(V_{1}\left(s_{k}\right) \cup V_{0}\left(s_{k}\right)\right)$ is formed by vertices not considered by the 3-median schema. The "do not care" label \# will be used to distinguish this condition. Observe that the number of medians is the same on schemata and structures, determining the name $p$-median schema (a condition that can be relaxed).

The same heuristic AH1 is then used to make the assignments, giving a clear and unique representation. The clusters identified in Figure 2 are $C_{1}\left(s_{k}\right)=\{1,8\}, C_{2}\left(s_{k}\right)=$ $\{4,5,9\}$, and $C_{3}\left(s_{k}\right)=\{3,7,10\}$.

The structure and schema $s_{k}$ defined above can also be used on the CPMP representation. The only modification is that now the clusters have capacities. Heuristic $\mathrm{AH} 2$ is the corresponding assignment heuristic in this case. Assume, for instance, that the cluster capacities are the same (a condition that can also be relaxed).

Each non-median vertex is assigned to its nearest median if the cluster capacity is not violated.

AH2

$$
\begin{aligned}
& \text { Read } s_{k} \text {, } \\
& Q \text {, } \\
& V_{1}\left(s_{k}\right)=\left\{\zeta_{1}, \zeta_{2}, \ldots, \zeta_{\left|V_{1}\left(s_{k}\right)\right|}\right\}, \\
& V_{0}\left(s_{k}\right)=\left\{\beta_{1}, \beta_{2}, \ldots, \beta_{\left|V_{0}\left(s_{k}\right)\right|}\right\} ; \\
& \alpha_{\beta_{j}}, j=1, \ldots,\left|V_{0}\left(s_{k}\right)\right| \text {, } \\
& \alpha_{\zeta_{i}}, i=1, \ldots,\left|V_{1}\left(s_{k}\right)\right| \text {, } \\
& \text { For } i=1 \text { to }\left|V_{1}\left(s_{k}\right)\right| \text { do } \\
& Q_{i}:=Q-\alpha_{\zeta_{i}} \text {, } \\
& \text { end_for } \\
& C_{i}\left(s_{k}\right):=\left\{\zeta_{i}\right\} ; \\
& r:=\operatorname{index}\left[\operatorname{Min}_{\left\{i=1, \ldots,\left|V_{1}\left(s_{k}\right)\right|\right\}}\left\{\mu_{\zeta_{i} \beta_{j}}\left|Q_{i}-\alpha_{\beta_{j}}\right| \geq 0\right\}\right] \text {; } \\
& Q_{r}:=Q_{r}-\alpha_{\beta_{j}} \text {, } \\
& C_{r}\left(s_{k}\right):=C_{r}\left(s_{k}\right) \cup\left\{\beta_{j}\right\} ;
\end{aligned}
$$

end_for

Define $X$ as the set of all structures and schemata that can be generated by the $0-1-\#$ string representation. For the PMP, the assignment heuristic AH1 allows completeness to $X$ in the sense that an optimal solution to the problem is always in $X$. The same is not true on heuristic AH2 for CPMP (capacity constraints may not be correctly represented).

The assignment heuristics can be defined in various ways, and the more elaborated they are, the better solutions they find to the clustering problems (although, generally increasing computational times).

\subsection{The Bi-Objective Optimization Problem}

The CGA is proposed to address the problem of evaluating schemata and structures in a common basis. While in the other evolutionary algorithms, evaluations of individuals are based on a single function (the fitness function), in CGA this process relies on two 
functions: mapping the space of structures and schemata onto $\Re_{+}$.

Let $X$ be the set of all structures and schemata that can be generated by the $0-1-\#$ string representation of Section 2.1, and consider two functions $f$ and $g$ defined as $f: X \rightarrow \Re_{+}$and $g: X \rightarrow \Re_{+}$such that $f\left(s_{k}\right) \leq g\left(s_{k}\right)$ for all $s_{k} \in X$. We define the double fitness evaluation of a structure or schema $s_{k}$, due to functions $f$ and $g$, as $f g$-fitness.

The CGA optimization problem implements the $f g$-fitness with the following two objectives:

1. Interval Minimization (Search for $s_{k} \in X$ of minimal $\left\{g\left(s_{k}\right)-f\left(s_{k}\right)\right\}$ )

This objective can be accomplished by schemata or structures. The response to the evolutionary search is given on a very adapted structure, then, a further optimization objective is needed to guide the search to find structures. The second objective is then:

2. $g$ Maximization (Search for $s_{k} \in X$ of maximal $g\left(s_{k}\right)$ )

Considering the schema representation, the $f g$-fitness evaluation increases as the number of labels \# decreases, and therefore structures have higher $f g$-fitness evaluation than schemata.

To attain this, a problem using CGA is modeled as the following bi-objective optimization problem (BOP):

$$
\begin{gathered}
\operatorname{Min}\left\{g\left(s_{k}\right)-f\left(s_{k}\right)\right\} \\
\operatorname{Max} g\left(s_{k}\right) \\
\text { subject to } g\left(s_{k}\right) \geq f\left(s_{k}\right), s_{k} \in X
\end{gathered}
$$

Functions $f$ and $g$ must be properly identified to represent the optimization objectives of the problems at issue. The $f g$-fitness process is particularized in the following for the clustering problems.

Consider a structure or schema $s_{k} \in X$. For PMP and CPMP, after the application of the assignment heuristics $\mathrm{AH} 1$ or $\mathrm{AH} 2, p=\left|V_{1}\left(s_{k}\right)\right|$ clusters $C_{i}\left(s_{k}\right)$ are identified corresponding to the median set $V_{1}\left(s_{k}\right)=\left\{\zeta_{1}, \zeta_{2}, \ldots, \zeta_{p}\right\}$.

Function $g$ is defined by $g\left(s_{k}\right)=\sum_{i=1}^{p} \sum_{j \in C_{i}\left(s_{k}\right)} \mu_{\zeta_{i} j}$, and function $f$ is defined by $f\left(s_{k}\right)=\sum_{i=1}^{p} \lambda_{i} \cdot\left[\left|C_{i}\left(s_{k}\right)\right|-1\right]$, where $\lambda_{i}=\operatorname{Min}_{j \in C_{i}\left(s_{k}\right)}\left\{\mu_{\zeta_{i} j}\right\}$ is the minimum cost (distance) assigned in cluster $i$, and $\left|C_{i}\left(s_{k}\right)\right|$ is the cardinality of the set $C_{i}\left(s_{k}\right)$.

For the schema $(1, \#, 0,0,0, \#, 0,0,1,1)$ represented in Figure 2 , we have $f(1, \#, 0,0,0, \#, 0,0,1,1)=2 * 1+1 * 2+3 * 2=10$ and $g(1, \#, 0,0,0, \#, 0,0,1,1)=$ $2+1+2+3+4=12$.

Clearly, $f\left(s_{k}\right) \leq\left(s_{k}\right)$ for all $s_{k} \in X$. The objective in BOP of minimizing the interval $g\left(s_{k}\right)-f\left(s_{k}\right)$ is directly related to the optimizing objectives on PMP and CPMP. The evaluation of function $f$ gives an "ideal" evaluation for the distances of assigned vertices in clusters, while function $g$ gives the actual evaluation for the distances of the assigned vertices. If $s_{k}$ is a structure, $g\left(s_{k}\right)$ gives the solution value for the respective problem, and the best (minimum) value is retained in the process.

\subsection{The Evolution Process}

The evolution process in CGA is conducted to accomplish interval minimization and $g$ maximization of the BOP. At the beginning of the process, the following two expected values are given to these objectives: 


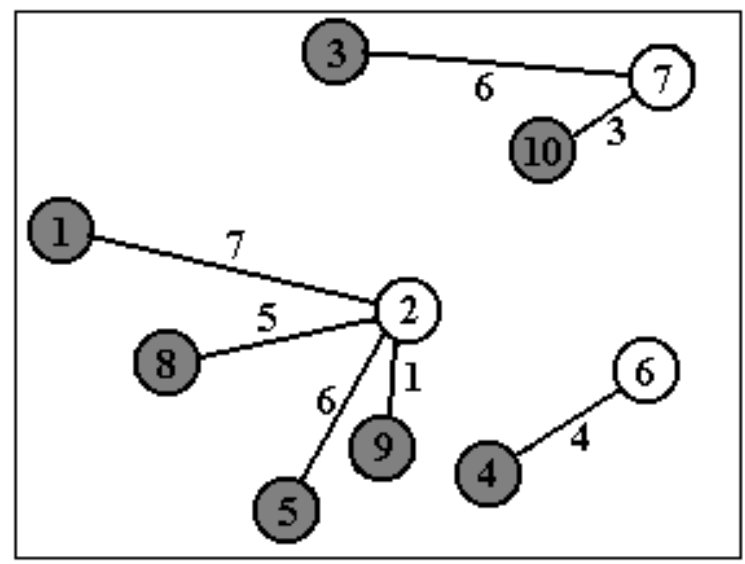

Figure 3: A random structure $(0,1,0,0,0,1,1,0,0,0)$.

\section{Expected Value to $g$ Maximization}

A non-negative real number $g_{\max }>\operatorname{Max}_{s_{k} \in X} g\left(s_{k}\right)$ that is an upper bound to $g\left(s_{k}\right)$ for each $s_{k} \in X$.

\section{Expected Value to Interval Minimization}

The interval length $d g_{\max }$, obtained from $g_{\max }$ using a real number $0 \leq d \leq 1$.

For the clustering problems, the overall bound $g_{\max }$ is obtained at the beginning of the CGA by generating a random complete structure and making $g_{\max }$ receive the $g$ evaluation for that structure.

For the 3-median example of Section 2.1, the random structure of Figure 3 gives $g_{\text {max }}=g(0,1,0,0,0,1,1,0,0,0)=32$, and for $d=0.1$, the expected interval length is $d g_{\max }=(0.1) .(32)=3.2$. In order to ensure that $g_{\max }$ is always an upper bound, after recombination, each new structure generated $s_{\text {new }}$ is rejected if $g_{\text {max }} \leq g\left(s_{\text {new }}\right)$.

The evolution process is then conducted considering an adaptive rejection threshold, which contemplates both objectives in BOP. Given a parameter $\alpha \geq 0$, the expression:

$$
g\left(s_{k}\right)-f\left(s_{k}\right) \geq d g_{\max }-\alpha . d\left[g_{\max }-g\left(s_{k}\right)\right]
$$

presents a condition for rejection from the current population of a schema or structure $s_{k}$.

The right hand side of Expression 1 is the threshold composed of the expected value to the interval minimization $d g_{\max }$ and the measure $\left[g_{\max }-g\left(s_{k}\right)\right]$, that shows the difference of $g\left(s_{k}\right)$ and $g_{\max }$ evaluations.

For $\alpha=0$, Expression 1 is equivalent to comparing the interval length obtained by $s_{k}$ and the expected length $d g_{\max }$. Schemata or structures are discarded if Expression 1 is satisfied. When $\alpha>0$, schemata are penalized and have a higher possibility of being discarded than structures, because structures present, in general, smaller differences $\left[g_{\max }-g\left(s_{k}\right)\right]$ than schemata.

Parameter $\alpha$ is related to time in the evolution process. Considering that the good schemata need to be preserved for recombination, the evolution parameter $\alpha$ starts from 
0 , and then increases slowly in small time intervals from generation to generation. The population at the evolution time $\alpha$, denoted by $P_{\alpha}$, is dynamic in size according to the value of the adaptive parameter $\alpha$ and can be emptied during the process.

The parameter $\alpha$ is now isolated in Expression 1, thus yielding the following expression and corresponding rank to $s_{k}$ :

$$
\alpha \geq \frac{d g_{\max }-\left[g\left(s_{k}\right)-f\left(s_{k}\right)\right]}{d\left[g_{\max }-g\left(s_{k}\right)\right]}
$$

\section{Rank}

The right hand side of Expression 2 gives a rank value to $s_{k}$ :

$$
\delta\left(s_{k}\right)=\frac{d g_{\max }-\left[g\left(s_{k}\right)-f\left(s_{k}\right)\right]}{d\left[g_{\max }-g\left(s_{k}\right)\right]}
$$

At the time they are created, structures and/or schemata receive their corresponding rank value $\delta\left(s_{k}\right)$. The rank of each schema or structure is compared to the current evolution parameter $\alpha$. At the moment a structure or schema is created, it is then possible to have some figure of its survivability. The higher the value of $\delta\left(s_{k}\right)$, the better the structure or schema to the BOP, and the greater the survival and recombination time.

Analyzing the ratio $\delta\left(s_{k}\right)$, it is possible to isolate the effects of $d$ and $g_{\max }$ in population size. It is clear that for small $d$, the population presents a slow increase and maintains a small dimension. A large $d$ presents the storage problems of a large population, but eventually with good structures. An undesired effect of $g_{\max }$ is that when $g_{\max } \gg g\left(s_{k}\right)$ for a large number of $s_{k} \in X$, they all have $\delta\left(s_{k}\right) \cong 1$, thus entailing the possibility of elimination from one generation to the next (after $\alpha=1$ ). Naturally, the effect can be an enormous reduction in population size in a few generations. Then the definition of parameters $d$ and $g_{\max }$ must be the result of a careful study.

\section{The CGA Operators}

In this section, we describe the operators that work on the evolution process in the CGA. The CGA initial population will be formed only by schemata. Recombination will create new schemata and structures.

Recombination can produce more adapted structures and/or schemata. The best structure needs to be kept in the process, generating a best feasible solution to the problem under consideration. Structures can be improved by mutation. After some generations, the population is composed of good schemata and probably good structures representing improved feasible solutions.

\subsection{Initial Population}

The initial population is composed exclusively of schemata, and for a sequence of generations, the population can increase by addition of new offspring generated out of the combination of two schemata.

Let $P_{0}$ be the initial population. For each schema, a proportion of random positions receives label 0 , and $p$ (number of clusters) random positions receive label 1 . The remaining positions receive label \#.

The schemata in $P_{0}$ will be (re)combined to produce offspring with some of the labels \# substituted by labels 1 or 0 seeking for structures. For the case of \# to 1 substitution, some original positions with label 1 are modified to 0 , maintaining the pre-fixed number of clusters. 
The proportion of labels \# has direct influence on the evolution process. Schemata with a small number of labels \# have a higher possibility of generating structures as offspring than those with large number of labels \#, but it is difficult to have good schemata generated at random. On the other side, schemata with large number of labels \# have small possibility of generating a structure as an offspring.

For the computational tests of Section 4, 20\% of $n$ random positions received label 0 , where $n$ is the size of $P_{0}$. The following can be an initial population for the 3-median example $(n=10)$ :

$$
\begin{aligned}
& s_{1}=(1,0, \#, 1,1, \#, 0, \#, \#, \#), s_{2}=(\#, 1,0,0, \#, 1, \#, \#, \#, 1), \\
& s_{3}=(\#, \#, 1,0, \#, 1,1, \#, \#, 0), s_{4}=(0, \#, \#, 1,0, \#, \#, 1, \#, 1), \\
& s_{5}=(1, \#, 0, \#, \#, 1,0,1, \#, \#), s_{6}=(\#, 0, \#, \#, 1,1,1,0, \#, \#) \\
& s_{7}=(\#, \#, \#, 0,1,0,1, \#, \#, 1), s_{8}=(\#, 1,0,0, \#, \#, 1,1, \#, \#) \\
& s_{9}=(0,0, \#, \#, \#, 1, \#, 1,1, \#), s_{10}=(0,1,0,1, \#, \#, \#, 1, \#, \#) .
\end{aligned}
$$

\subsection{Recombination}

We have two purposes in the evolution process: to obtain solutions to the $g$ maximization objective on the BOP and that these structures be the best solutions to the interval minimization problem on the BOP. The selection of structures and/or schemata for recombination will be conducted to attain these two objectives. The first one is attained by selecting for recombination schemata with a small number of labels \#, and the second considering structures or schemata with small $d_{k}=\frac{g\left(s_{k}\right)-f\left(s_{k}\right)}{g\left(s_{k}\right)}$.

The structures and schemata in population $P_{\alpha}$ are maintained on ascending order, according to the key $\Delta\left(s_{k}\right)=\frac{1+d_{k}}{\left|V_{1}\left(s_{k}\right)\right|+\left|V_{0}\left(s_{k}\right)\right|}$. Structures $\left(V_{\#}\left(s_{k}\right)=\emptyset\right)$, schemata with $\left|V_{\#}\left(s_{k}\right)\right|$ small, and structures and/or schemata presenting small $d_{k}$ are better and appear in the initial positions.

Two structures and/or schemata are selected for combination. The first is called the base ( $\left.s_{\text {base }}\right)$ and is randomly selected out of the $n$ first positions in $P_{\alpha}$; in general, it is a good structure or a good schema ( $d_{\text {base }}$ is small). If it turns out to be a schema, the following recombination process tries to preserve labels 1 or 0 already assigned in $s_{\text {base }}$. The second structure or schema is called the guide $\left(s_{\text {guide }}\right)$ and is randomly selected out of the total population. The objective of the $s_{\text {guide }}$ selection is the conduction of a guided modification on $s_{\text {base }}$. The current labels in corresponding positions are compared. Let $s_{\text {new }}$ be the new structure or schema (offspring) after recombination. order):

Structure or schema $s_{n e w}$ is obtained by applying the following operations (in this

\section{\{Recombination $\}$}

(i) For each $j \in\{1, \ldots, n\}$ presenting $s_{\text {basej }}=\#$ and $s_{\text {guidej }}=\#$ set $s_{\text {new }}=\#$;

(ii) For each $j \in\{1, \ldots, n\}$ presenting $s_{\text {basej }}=1$ and $s_{\text {guide }}=1$ set $s_{\text {new }}=1$;

(iii) For each $j \in\{1, \ldots, n\}$ presenting $s_{\text {base }}=0$ and $s_{\text {guide } j}=0$ set $s_{\text {new }}=0$;

(iv) For each $j \in\{1, \ldots, n\}$ presenting $s_{\text {base }}=1$ and $s_{\text {guidej }}=\#$ set $s_{\text {new }}=1$;

(v) For each $j \in\{1, \ldots, n\}$ presenting $s_{\text {basej }}=0$ and $s_{\text {guidej }}=\#$ set $s_{\text {new }}=0$;

(vi) For each $j \in\{1, \ldots, n\}$ presenting $s_{\text {basej }}=\#$ and $s_{\text {guidej }}=0$ set $s_{\text {newj }}=0$;

(vii) For each $j \in\{1, \ldots, n\}$ presenting $s_{\text {basej }}=\#$ or 0 and $s_{\text {guide } j}=1$ then two cases are possible:

1. set $s_{\text {new }}=1$, also if $\left|V_{1}\left(s_{\text {new }}\right)\right|=p$, set $s_{\text {newl }}=0$ for $l \in\{1, \ldots, n\}$ presenting $s_{\text {newl }}=1$ (randomly selected), 
2. set $s_{\text {new }}=1$, also if $\left|V_{1}\left(s_{\text {new }}\right)\right|=p$, set $s_{\text {newl }}=0$ for each $l \in\{1, \ldots, n\}$ presenting $s_{\text {newl }}=1$ generating $p$ new structures and/or schemata,

(viii) For each $j \in\{1, \ldots, n\}$ presenting $s_{\text {basej }}=1$ and $s_{\text {guidej }}=0$ then two cases are possible:

1. set $s_{\text {new }}=0$ and $s_{\text {newl }}=1$ for $l \in\{1, \ldots, n\}$ presenting $s_{\text {newl }}=0$ (randomly selected),

2. set $s_{\text {newj }}=0$ and $s_{\text {newl }}=1$ for each $l \in\{1, \ldots, n\}$ presenting $s_{\text {newl }}=0$ generating $\left|V_{0}\left(s_{\text {base }}\right)\right|$ new structures and/or schemata.

For each offspring generated, operations (i)-(v) preserve in $s_{n e w}$ the labels present in $s_{\text {base }}$ (schema information). Operations (vi)-(viii) produce different offspring. Operation (vi) produces one $s_{n e w}$ that is $s_{\text {base }}$ with some additional labels 0 . Operations (vii) and (viii) can be seen as a mutation operator generating in the second case, $p$ or $\left|V_{0}\left(s_{\text {base }}\right)\right|$ new offspring. These mutation-like phases on recombination (operations (vii) and (viii)) are imposed by the number of clusters in $s_{\text {new }}$ (the number of 1 's is fixed on schemata and structures).

When $s_{\text {base }}$ is a structure $\left(V_{\#}\left(s_{\text {base }}\right)=\emptyset\right)$, the following interchange heuristic is performed as a local search mutation:

\section{IH $\{$ Interchange Heuristic $\}$}

For each $j \in V_{1}\left(s_{\text {base }}\right)$ do

For each $l \in V_{0}\left(s_{\text {base }}\right)$ do

Interchange $j$ and $l$ and generating an offspring $s_{\text {new }}$; \{offspring generation

Interchange $l$ and $j$;

\section{End_for}

End_for

The application of the local search mutation IH produces $p .\left|V_{0}\left(s_{\text {base }}\right)\right|$ new structures. For each $\alpha$ value, $n$ new structures and/or schemata are generated, their ranks computed and compared with $\alpha$, and included or not in the new population.

\subsection{The Algorithm}

The constructive genetic algorithm (CGA) can be summed up by the pseudocode:

\section{CGA \{Constructive Genetic Algorithm}

Given $g_{\max }$ and $d$;

$\alpha:=0$;

$\epsilon:=0.05$;

Initialize $P_{\alpha}$;

Evaluate $P_{\alpha}$;

For all $s_{k} \in P_{\alpha}$ compute $\delta\left(s_{k}\right)$

end_for

While (not stop condition) do

For all $s_{k} \in P_{\alpha}$ satisfying $\alpha<\delta\left(s_{k}\right)$ do

$$
\alpha:=\alpha+\epsilon
$$

\{ time interval \}

$\{$ initial population $\}$

$\{f g$-fitness $\}$

$\{$ rank computation $\}$

$\{$ evolution test $\}$ 
Select $P_{\alpha}$ from $P_{\alpha-\epsilon}$;

Recombine $P_{\alpha}$;

Evaluate $P_{\alpha}$;

end_for

For all new $s_{k} \in P_{\alpha}$ compute $\delta\left(s_{k}\right)$

end_for

end_while

The CGA algorithm begins with the recombination procedures (schemata) and no local search mutation. After a sequence of generations, the number of structures increases and so does the consequent application of IH mutations. At final generations, only IH mutations are performed.

Our stop conditions occur with an emptied population (assured by a sufficiently higher $\alpha$ ) or at a predefined number of generations. The population increases, after the initial generations, reaching an upper limit (in general controlled by storage conditions) and decreases for higher values of the evolution parameter $\alpha$ (see Figure 4). The structure corresponding to the best problem solution must be kept in the process.

\section{Computational Results}

The CGA was initially tested using a partial sample of the p-median data drawn from the OR-library (Beasley, 1990). The sizes are 100, 200, 300, 400, and 500 vertices. The number of medians varies from 5 to 67 .

The computational tests are reported in Table 1. The Lagrangian heuristic results appeared in Senne and Lorena (2000); they used local search and an iterative locationallocation procedure to obtain feasible solutions. It was programmed in $\mathrm{C}$, running on an IBM Risc/6000 model 3AT workstation (compiled using xlc compiler with -O2 optimization option). The CGA feasible solution is the $g\left(s_{k}\right)$ evaluation for the best structure kept, using the simple AH1 heuristic for the assignments. The gap is calculated as [(Feasible Solution-Optimal solution) $\left.{ }^{*} 100\right] /($ Feasible Solution).

The CGA results are slightly worse than the Lagrangian ones, but all gaps are inferior to $0.73 \%$ and null for nine instances. For all the CGA results presented in this section, the algorithm was coded in C, running on a Pentium 166 Mhz. The computational times (Table 1) for both algorithms are not comparable due to the use of different machines, although the IBM Risc/6000 could be considered faster than a Pentium 166 Mhz.

It can be seen in Table 1 that a large number of medians increase the CGA computational times. This is easy to explain as the local search mutation is used more intensively in these cases. For all CGA results presented in this section, $d=0.1$, and the evolution control (time interval) used is: $\epsilon=0.05$ for $0 \leq \alpha \leq 1$ and $\epsilon=0.025$ for $\alpha>1$.

In the following figures, we examine the running test with problem pmed1. Figure 4 shows the population evolution by generation. A maximum population size is obtained at generation $25\left(P_{1.25}\right)$ with approximately 2000 structures and/or schemata. The population decreases after $\alpha=1.25$, showing that the number of new structures and/or schemata by generation is smaller than the number that do not pass the evolution test. The procedure stopped with an empty population at $\alpha=4.5$.

Figure 5 shows the maximum number of vertices for each structure or schema generated out of recombination and local search mutation. A structure is obtained only after generation 13. Even in a population mostly of structures, schemata form

Evolutionary Computation Volume 9, Number 3 


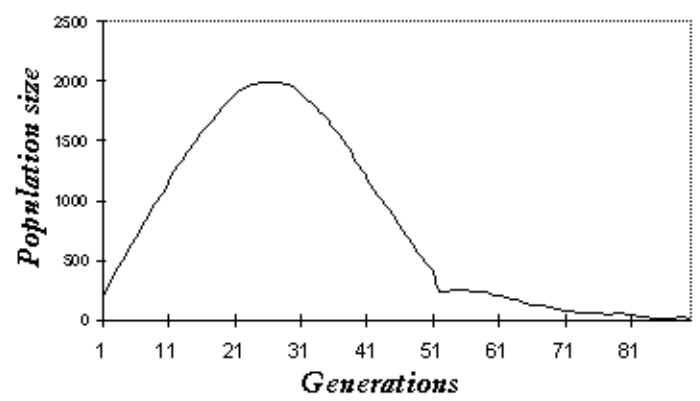

Figure 4: Population size by generation.

Table 1: Computational results (OR-library instances).

\begin{tabular}{|l|l|l|l|l|l|c|c|}
\hline Problem & $\begin{array}{l}\text { Vertices } \\
(\mathrm{n})\end{array}$ & $\begin{array}{l}\text { Medians } \\
(\mathrm{p})\end{array}$ & $\begin{array}{l}\text { Optimal } \\
\text { Solution }\end{array}$ & $\begin{array}{l}\text { Lagrangian } \\
\text { heuristic } \\
\text { gap }(\%)\end{array}$ & $\begin{array}{l}\text { Lagrangian } \\
\text { heuristic } \\
\text { times(sec. })\end{array}$ & $\begin{array}{l}\text { CGA } \\
\text { gap } \\
(\%)\end{array}$ & $\begin{array}{c}\text { CGA } \\
\text { times } \\
(\text { sec.) }\end{array}$ \\
\hline pmed1 & 100 & 5 & 5819 & 0 & 0.93 & 0 & 28 \\
pmed2 & 100 & 10 & 4093 & 0 & 1.34 & 0 & 37 \\
pmed3 & 100 & 10 & 4250 & 0 & 1.79 & 0 & 34 \\
pmed4 & 100 & 20 & 3034 & 0 & 1.58 & 0 & 230 \\
pmed5 & 100 & 33 & 1355 & 0 & 2.07 & 0.36 & 375 \\
pmed6 & 200 & 5 & 7824 & 0 & 4.75 & 0 & 172 \\
pmed7 & 200 & 10 & 5631 & 0 & 5.45 & 0 & 238 \\
pmed8 & 200 & 20 & 4445 & 0 & 5.03 & 0.20 & 1055 \\
pmed9 & 200 & 40 & 2734 & 0 & 10.6 & 0.73 & 3331 \\
pmed10 & 200 & 67 & 1255 & 0 & 17.4 & 0.15 & 4325 \\
pmed11 & 300 & 5 & 7696 & 0 & 10.0 & 0 & 369 \\
pmed12 & 300 & 10 & 6634 & 0 & 11.7 & 0.04 & 677 \\
pmed13 & 400 & 5 & 8162 & 0.012 & 19.11 & 0 & 555 \\
pmed14 & 500 & 5 & 9138 & 0 & 20.60 & 0 & 1875 \\
\hline
\end{tabular}

a representative part of it; after all, a schema may receive a better $f g$-fitness than a structure.

Figure 6 shows the improvements on the best $d_{k}$ by generation, and finally, Figure 7 shows the best $g$ function evaluation for a structure (p-median solution) at each generation.

A classical mathematical programming formulation for $\mathrm{p}$-median problems has been considered (Beasley, 1985, 1993; Christofides and Beasley, 1982; Senne and Lorena, 2000). Considering a primal-dual approach, the set of OR-Library p-median instances can be considered that is composed of easy problems in the sense of duality gaps. The gaps can all be closed using a Lagrangian (or Lagrangian/surrogate) approach (Beasley, 


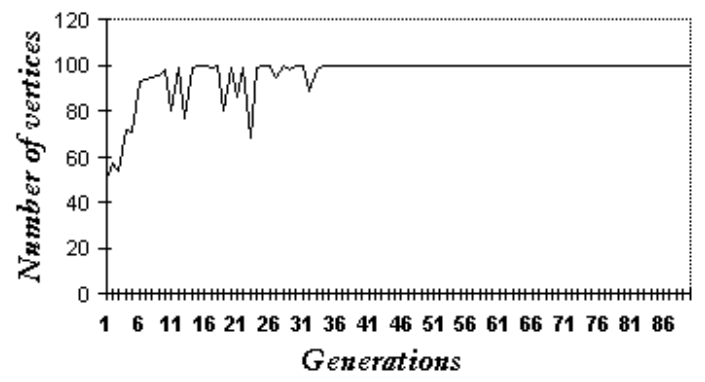

Figure 5: Maximum number of vertices by generation.

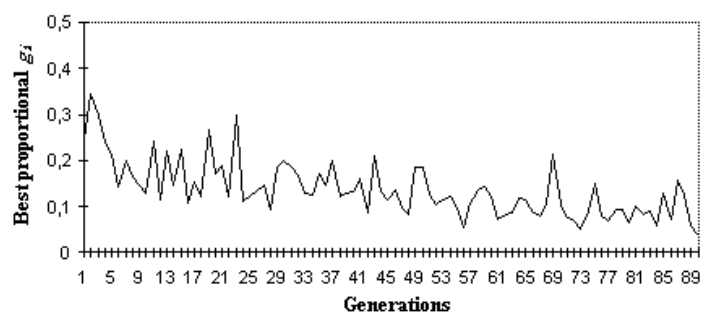

Figure 6: Best $d_{k}$ by generation.

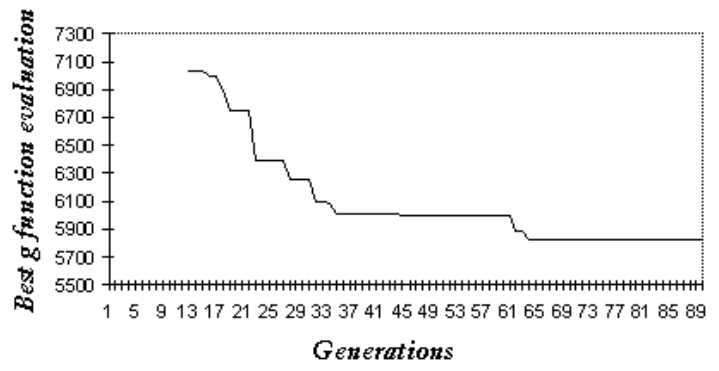

Figure 7: Best $g$ evaluation by generation.

1993; Senne and Lorena, 2000). Based on that, another test suit was performed with another set of instances (Galvão and ReVelle, 1996; Senne and Lorena, 2000) that presents large duality gaps for some values of $p$. A comparison was also made with the direct application of the interchange heuristic IH on a set of structures.

Table 2 shows the computational experiments. The mean CGA gaps for 5 replica- 
Table 2: Problems presenting large duality gaps.

\begin{tabular}{||c||c||c||c||c||c||c|}
\hline $\begin{array}{c}\text { Number of } \\
\text { vertices } \\
(\mathrm{n})\end{array}$ & $\begin{array}{c}\text { Number of } \\
\text { medians } \\
(\mathrm{p})\end{array}$ & $\begin{array}{c}\text { Duality } \\
\text { gap } \\
(\%)\end{array}$ & $\begin{array}{c}\text { Optimal } \\
\text { solution }\end{array}$ & $\begin{array}{c}\text { Lagrangian } \\
\text { heuristic } \\
\text { gap }(\%)\end{array}$ & $\begin{array}{c}\text { IH } \\
\text { gap } \\
(\%)\end{array}$ & $\begin{array}{c}\text { CGA } \\
\text { gap } \\
(\%)\end{array}$ \\
\hline 100 & 5 & 0.346 & 5703 & 0 & 6.36 & 0 \\
100 & 10 & 3.728 & 4426 & 2.553 & 10.72 & 0 \\
100 & 15 & 0.895 & 3893 & 0.745 & 8.20 & 0.136 \\
100 & 20 & 0.093 & 3565 & 0.084 & 6.07 & 0.299 \\
100 & 25 & 0.067 & 3291 & 0 & 4.10 & 0.243 \\
100 & 30 & 0.056 & 3032 & 0.066 & 3.73 & 0.425 \\
100 & 40 & 0 & 2542 & 0 & 3.57 & 0.432 \\
100 & 50 & 0 & 2083 & 0 & 4.11 & 0.096 \\
150 & 5 & 1.404 & 10839 & 0 & 4.24 & 0 \\
150 & 10 & 3.158 & 8729 & 0.252 & 8.21 & 0 \\
150 & 15 & 4.906 & 7390 & 0.731 & 12.15 & 0.184 \\
150 & 20 & 2.975 & 6454 & 3.595 & 14.88 & 0.547 \\
150 & 25 & 1.009 & 5875 & 2.060 & 13.20 & 0.204 \\
150 & 30 & 0.208 & 5495 & 0.564 & 12.59 & 0.345 \\
150 & 40 & 0.068 & 4907 & 0.143 & 8.40 & 0.346 \\
150 & 50 & 0.062 & 4374 & 0 & 6.24 & 0.137 \\
\hline
\end{tabular}

tions are lower than $0.547 \%$ for all instances. The CGA results compare favorably with the Lagrangian ones. The Lagrangian heuristic's worst result is $3.595 \%$, and for five instances, the Lagrangian heuristic gap is greater than $0.547 \%$. To apply the $\mathrm{IH}$ alone, we start with an initial set of $n$ structures, then $\mathrm{IH}$ is applied on each structure. The process is replicated with 5 initial randomly generated sets, and the best solution reported in Table 2. The direct application of $\mathrm{IH}$ yields gaps varying from $3.57 \%$ to $14.88 \%$. The comparison is included to accentuate the concept that CGA works with a population that retains adapted structures after the constructive phase.

The CGA was also tested on instances of the CPMP. Two sets of 10 instances are considered with (50x5) and (100x10) vertices and medians, respectively. They appeared in Osman and Christofides (1994). The results are shown in Table 3, where columns are composed of the problem identification, the best-known solution, results for some heuristics (H.OC, H1+F1, H1+B1, HSS.OC, HSS.C and TS1+FBA) used in Osman and Christofides (1994), and in the last column, the CGA solution.

Heuristic H.OC is a simple constructive heuristic, while $\mathrm{H} 1+\mathrm{F} 1$ and $\mathrm{H} 1+\mathrm{B} 1$ begin with the H.OC solution and make some permutations using "first improve" and "best improve" strategies. Algorithm TS1+FBA is a tabu search implementation. Simulated annealing is implemented with algorithms HSS.OC and HSS.C, which use distinct cooling schedules. The best results are obtained using the algorithm HSS.OC, where the simulated annealing probabilistic acceptance was improved in three ways. It makes use of a non-monotonic cooling schedule, a systematic neighborhood search, and a termination condition based on the number of temperature resets performed without improving the best solution.

Table 4 presents the gaps to the best solution reached by the 7 heuristics, as well as the times for heuristic HSS.OC and the mean times for 5 replications of the CGA. 
Table 3: Problem CPMP-CGA and Osman and Christofides results.

\begin{tabular}{|l|l|l|l|l|l|l|l|r|r|r|}
\hline Problem & Vertices & Medians & $\begin{array}{l}\text { Best } \\
\text { solution }\end{array}$ & H.OC & H1+F1 & H1+B1 & HSS.OC & HSS.C & $\begin{array}{r}\text { TS1+ } \\
\text { FBA }\end{array}$ & CGA \\
\hline 1 & 50 & 5 & 713 & 786 & 780 & 818 & 713 & 734 & 734 & 713 \\
2 & 50 & 5 & 740 & 816 & 762 & 778 & 740 & 740 & 740 & 740 \\
3 & 50 & 5 & 751 & 972 & 811 & 816 & 751 & 751 & 751 & 751 \\
4 & 50 & 5 & 651 & 891 & 651 & 652 & 651 & 651 & 651 & 651 \\
5 & 50 & 5 & 664 & 804 & 746 & 677 & 664 & 664 & 664 & 664 \\
6 & 50 & 5 & 778 & 882 & 841 & 847 & 778 & 778 & 778 & 778 \\
7 & 50 & 5 & 787 & 968 & 852 & 824 & 787 & 805 & 787 & 787 \\
8 & 50 & 5 & 820 & 945 & 834 & 837 & 820 & 820 & 821 & 826 \\
9 & 50 & 5 & 715 & 752 & 735 & 734 & 715 & 715 & 715 & 715 \\
10 & 50 & 5 & 829 & 1017 & 844 & 891 & 829 & 829 & 829 & 834 \\
11 & 100 & 10 & 1006 & 1761 & 1020 & 1019 & 1006 & 1006 & 1009 & 1014 \\
12 & 100 & 10 & 966 & 1567 & 1004 & 974 & 966 & 966 & 968 & 969 \\
13 & 100 & 10 & 1026 & 1847 & 1144 & 1053 & 1026 & 1026 & 1026 & 1026 \\
14 & 100 & 10 & 982 & 1635 & 998 & 1054 & 985 & 982 & 985 & 987 \\
15 & 100 & 10 & 1091 & 1517 & 1098 & 1138 & 1091 & 1091 & 1096 & 1091 \\
16 & 100 & 10 & 954 & 1780 & 1063 & 993 & 954 & 954 & 957 & 955 \\
17 & 100 & 10 & 1034 & 1665 & 1104 & 1092 & 1039 & 1037 & 1040 & 1034 \\
18 & 100 & 10 & 1043 & 1345 & 1089 & 1136 & 1045 & 1045 & 1045 & 1045 \\
19 & 100 & 10 & 1031 & 1634 & 1105 & 1125 & 1031 & 1032 & 1034 & 1032 \\
20 & 100 & 10 & 1005 & 1872 & 1036 & 1030 & 1005 & 1019 & 1005 & 1039 \\
\hline
\end{tabular}

The times for HSS.OC are reported in Maniezzo et al. (1998), where the authors have implemented the heuristic in Fortran 77, also running on a Pentium 166 Mhz. Tables 3 and 4 show that the CGA results were as good as TS1+FBA and little worse than HSS.OC and HSS.C (i.e., their best results are comparable).

The CGA results can be considered good, spending small computer times. Considering effective implementations of Lagrangian relaxation heuristics and simulated annealing, the CGA results are comparable, and even better in some cases.

\section{Conclusion}

This work describes a constructive approach to genetic algorithms and an application to some clustering problems in graphs.

The CGA provides some new features to genetic algorithms. The principal CGA characteristics can be summarized as:

1. the direct work and evaluation of schemata,

2. the population is dynamic in size,

3. the initial population is formed by schemata,

4. the new $f g$-fitness provides double evaluation of schemata and structures,

5. at the creation time schemata and structures receive a rank, 
Table 4: Problem CPMP:CGA and Osman and Christofides gaps to the best solution.

\begin{tabular}{||c||c||c||c||c||c||c||c||c||c|}
\hline Problem & H.OC & H1+FI & H1+BI & HSS.OC & HSS.C & TS1+FBA & $\begin{array}{c}\text { HSS.OC } \\
\text { times } \\
\text { (sec.) }\end{array}$ & CGA & $\begin{array}{c}\text { CGA } \\
\text { times } \\
\text { (sec.) }\end{array}$ \\
\hline 1 & 10.23 & 9.39 & 14.72 & 0 & 2.94 & 2.94 & 4.34 & 0 & 2 \\
2 & 10.27 & 2.97 & 5.13 & 0 & 0 & 0 & 3.94 & 0 & 2 \\
3 & 29.42 & 7.98 & 8.65 & 0 & 0 & 0 & 4.10 & 0 & 12 \\
4 & 36.86 & 0 & 0.15 & 0 & 0 & 0 & 3.39 & 0 & 2 \\
5 & 21.08 & 12.34 & 1.95 & 0 & 0 & 0 & 3.77 & 0 & 8 \\
6 & 13.36 & 8.09 & 8.86 & 0 & 0 & 0 & 5.52 & 0 & 2 \\
7 & 22.99 & 8.25 & 4.70 & 0 & 2.28 & 0 & 5.15 & 0 & 3 \\
8 & 15.24 & 1.70 & 2.07 & 0 & 0 & 0.12 & 10.18 & 0.73 & 11 \\
9 & 5.17 & 2.79 & 2.65 & 0 & 0 & 0 & 11.43 & 0 & 2 \\
10 & 22.67 & 1.80 & 7.47 & 0 & 0 & 0 & 3.40 & 1.44 & 14 \\
11 & 75.04 & 1.39 & 1.29 & 0 & 0 & 0.29 & 100.57 & 0.79 & 614 \\
12 & 62.21 & 3.93 & 0.82 & 0 & 0 & 0.20 & 128.67 & 0.31 & 296 \\
13 & 80.01 & 11.50 & 2.63 & 0 & 0 & 0 & 106.13 & 0 & 327 \\
14 & 66.49 & 1.62 & 7.33 & 0.30 & 0 & 0.30 & 74.36 & 0.50 & 303 \\
15 & 38.91 & 0.54 & 4.21 & 0 & 0 & 0.36 & 129.85 & 0 & 315 \\
16 & 86.58 & 11.42 & 4.08 & 0 & 0 & 0.31 & 136.88 & 0.10 & 271 \\
17 & 61.02 & 6.76 & 5.60 & 0.48 & 0.29 & 0.58 & 122.47 & 0 & 332 \\
18 & 28.95 & 4.41 & 8.91 & 0.19 & 0.19 & 0.19 & 94.73 & 0.19 & 380 \\
19 & 58.48 & 7.17 & 9.11 & 0 & 0.09 & 0.29 & 82.87 & 0.09 & 410 \\
20 & 86.26 & 3.08 & 2.48 & 0 & 1.39 & 0 & 70.60 & 3.38 & 503 \\
\hline
\end{tabular}

6. good schemata and structures have high ranks and greater surviving and recombination times,

7. recombination preserves good schemata,

8. local search mutation to structures provides population diversification,

9. one objective of the CGA is the construction of a population with good structures after some generations.

For the clustering problems studied (PMP and CPMP), assignment heuristics decode common binary representations. The (good) effects of the heuristics are reflected to the whole population. The same CGA representation (composed with an assignment heuristic) was also tested (and described in an earlier version of this paper) on the Min Cut Clustering Problem considered in Johnson and Mehrotra (1992). It is the problem of partitioning the vertex set of a given graph into a pre-fixed number of clusters such that the sum of the cluster vertex weights have inferior and superior limits, while the sum of the clusters edge weights is maximized (or, alternatively, the sum of edge weights outside the clusters is minimized).

These representations and assignment decoders can be used in other clustering problems, but it is important to note that the CGA concepts and properties are independent on the representation and decoders used. Some early results used different 
representations of the CGA application to 2D-cutting (Lorena and Lopes, 1996) and k-coloring (Ribeiro and Lorena, 1997) problems.

Some complementary research can be made on experimental parameter design $(\epsilon$ and $d$ ), additional representation decoders (heuristics), and definitions of functions $f$ and $g$ and of parameter $g_{\max }$. It is expected that the good behavior obtained with the clustering problems studied here can be repeated with other difficult optimization problems.

\section{Acknowledgments}

The first author acknowledges Conselho Nacional de Desenvolvimento Cientfico e Tecnolgico - CNPq (proc. 350034/91-5, 520844/96-3, 680082/95-6) and Fundação para o Amparo a Pesquisa no Estado de S. Paulo - FAPESP (proc. 95/9522-0 e 96/04585-6) for partial financial support. We acknowledge the comments and suggestions of two anonymous referees and the suggestions for text improvement made by the editor, Professor D. Whitley, and Professor P. P. B. Oliveira from UNIVAP-Universidade do Vale do Paraíba.

\section{References}

Bäck, T. and Schwefel, H.-P. (1993). An Overview of evolutionary algorithms for parameter optimization. Evolutionary Computation, 1(1):1-23.

Beasley, J. E. (1985). A note on solving large p-median problems. European Journal of Operational Research, 21:270-273.

Beasley, J. E. (1990). OR-Library: distribution test problems by electronic mail. Journal of the Operational Research Society, 41:1069-1072.

Beasley, J. E. (1993). Lagrangian heuristics for Location problems. European Journal of Operational Research, 65:383-399.

Christofides, N. and Beasley, J. E. (1982). A tree search algorithm for the p-median problems. European Journal of Operational Research, 10:196-204.

Davis, L. D. (1991). Handbook of Genetic Algorithms. Van Nostrand Reinhold, New York, New York.

De Jong, K. (1975). An analysis of the behavior of a class of genetic adaptive systems. Ph.D. thesis, Computer Science Department, University of Michigan, Ann Arbor, Michigan.

Densham, P. J. and Rushton, G. (1992). A more efficient heuristic for solving large P-median problems. Papers in Regional Science, 71:307-329.

Efroymson, M. A. and Ray, T. L. (1966). A branch-and-bound algorithm for plant location. Operations Research, 14:361-368.

Galvão, R. D. and Raggi, L. A. (1989). A method for solving to optimality uncapacitated location problems. Annals of Operations Research, 18:225-244.

Galvão, R. D. and ReVelle, C. S. (1996). A Lagrangian heuristic for the maximal covering location problem. European Journal of Operational Research, 18:114-123.

Garey, M. R. and Johnson, D. S. (1979). Computers and Intractability: a Guide to the Theory of NPCompleteness. Freeman, San Francisco, California.

Goldberg, D. E. (1989). Genetic algorithms in search, optimization and machine learning, pages 11-172, Addison-Wesley, Reading, Massachusetts.

Goldberg, D. E., Korb, B., and Deb, K. (1989). Messy genetic algorithms: Motivation, analysis, and first results. Complex Systems, 3:493-530. 
Goldberg, D. E., Deb, K., Kargupta, H., and Harik, G. (1993). Rapid, accurate optimization of difficult problems using fast messy genetic algorithms. IlliGAL Report No. 93004, Illinois Genetic Algorithms Laboratory, Department of General Engineering, University of Illinois, Urbana, Illinois.

Goodchild, M. F. and Noronha, V. (1983). Location-allocation for small computers. Monograph No. 8, Department of Geography, University of Iowa, Iowa City, Iowa.

Hakimi, S. L. (1964). Optimum distribution of switching centers and the absolute centers and the medians of a graph. Operations Research, 12:450-459.

Hakimi, S. L. (1965). Optimum distribution of switching centers in a communication network and some related graph theoretic problems. Operations Research, 13:462-475.

Holland, J. H. (1975). Adaptation in natural and artificial systems, pages 11-147, MIT Press, Cambridge, Massachusetts.

Jarvinen, P., Rajala, J., and Sinervo, H. (1972). A branch and bound algorithm for seeking the p-median. Operations Research, 20:173-178.

Johnson, E. L. and Mehrotra, A. (1992). Min-Cut Clustering. Working paper, School of Industrial \& Systems Engineering, Georgia Institute of Technology, Atlanta, Georgia.

Kargupta, H. (1995). Search, Polynomial Complexity, and The Fast Messy Genetic Algorithm. IlliGAL Report No. 95008, Illinois Genetic Algorithms Laboratory, Department of General Engineering, University of Illinois, Urbana, Illinois.

Klein, K. and Aronson, J. E. (1991). Optimal clustering: a model and method. Naval Research Logistics, 38:447-461.

Lorena, L. A. N. and Lopes, F. B. (1996). A dynamic list heuristic for 2D-cutting. In Dolezal, J. and Fidler, J., editors, System Modeling and Optimization, pages 481-488, Chapman-Hall, London, UK.

Lorena, L. A. N. and Lopes, L. S. (1996). Computational experiments with genetic algorithms applied to set covering problems. Pesquisa Operacional, 16:41-53.

Lorena, L. A. N. and Lopes, L. S. (1997). Genetic algorithms applied to computationally difficult set covering problems. Journal of the Operational Research Society, 48:440-445.

Maniezzo, V., Mingozzi, A., and Baldaci, R. (1998). A bionomic approach to the capacitated pmedian problem. Journal of Heuristics, 4(3):262-280.

Michalewicz, Z. (1996). Genetic Algorithms + Data Structures = Evolution Programs. Springer-Verlag, Berlin, Germany.

Mitchell, M. (1996). An Introduction to Genetic Algorithms. MIT Press, Cambridge, Massachusetts.

Mulvey, J. M. and Beck, M. P. (1984). Solving capacitated clustering problems. European Journal of Operational Research, 18:339-348.

Neebe, A. W. (1978). A branch and bound algorithm for the p-median transportation problem. Journal of the Operational Research Society, 29:989-995.

Osman, I. H. and Christofides, N. (1994). Capacitated clustering problems by hybrid simulated annealing and tabu search. International Transactions in Operational Research, 1(3):317-336.

Ribeiro Filho, G. and Lorena, L. A. N. (1997). A constructive genetic algorithm for graph coloring. In Kumar, S. and Sniedovich, M., editors, 1997 Asia-Pacific Operations Research Societies Conference, page 113, Australian Society for Operations Research, Melbourne, Australia.

Rolland, E., Schilling, D. A., and Current, J. R. (1997). An efficient Tabu search procedure for the p-median problem. European Journal of Operational Research, 96:329-342. 
Rosing, K. E. and ReVelle, C. S. (1997). Heuristic concentration: Two stage solution construction. European Journal of Operational Research, 97:75-86.

Rosing, K. E., ReVelle, C. S., Schilling, D. A., and Current, J. R. (1998). Heuristic concentration and Tabu search: A head to head comparison. European Journal of Operational Research, 104(1):9399.

Senne, L. F. and Lorena L. A. N. (2000). Lagrangian/Surrogate Heuristics for p-Median Problems. In Laguna, M. and Gonzalez-Velarde, J. L., editors, Computing Tools for Modeling, Optimization and Simulation: Interfaces in Computer Science and Operations Research, pages 115-130, Kluwer Academic Publishers, London, UK.

Spath, H. (1980). Cluster Analysis Algorithms for data reduction and classification of objects. Ellis Horwood Publishers, New York, New York.

Teitz, M. B. and Bart, P. (1968). Heuristic methods for estimating the vertex median of a weighted graph. Operations Research, 16:955-961.

Zupan, J. (1982). Clustering of Large Data Sets. John Wiley and Sons, New York, New York. 